"Journal für die reine und angewandto Mathematik," Bd. cxrv., Heft 4; Berlin, 1895.

"Annals of Mathematics," Vol. Ix., No. 2, January, 1895; University of Virginiil.

"Indian Engiueering," Vol. xvrr.; Nos. 8, 9, 10.

A New Theorem in Probability. By Rev. T. C. Srumons, M.A. Read April 4th, 1895. Received, in revised form, June 6th, 1895.

1. "If an event happen on the average once in $\mathrm{m}$ times, $\mathrm{m}$ being greater than unity, then it is nore likely to happen less thun once in $\mathrm{m}$ times than it is to huppen more than muce in $\mathrm{m}$ times." In the present paper I undertake to prove this novel proposition, which may loe enunciated more explicitly thus:- "If an event may happen in $b$ ways and fail in a ways, a heing greater than $b$, and all these ways are equally likely to occur, then, $\mu$ trials being madc, where $\mu$ is any multiple of $a+b$, linge or' small, or any ranulom number, the event is more likely to) happen less than $\frac{\mu b}{a+b}$ times than it is to happen more than $\frac{\mu b}{a+b}$ times." Noreover, if the ratio of $a$ to $b$ be greater than 4 , I shall ventne to assert and prove a wider proposition, viz., that the event is more likely than mot to happen less than $\frac{\mu b}{a+b}$ times. 'T'his amounts to) suying that if a die, for justance, be tlirown any number of times, liuge or surall, chosen at ranclom, the number of appearances of the ace is more likcly than not to he less than $\frac{1}{6}$ of the number of throws. For reasous which will bo stated in A A.t. 32, $1 \mathrm{am}$ compelled at present to (qualify the foregoing statements by the linitation that $b=1$.

2. 'I'he lirst sugrgestion of such a proposition arose in this way. At the heriming of the present year J was engaged, for a purpose to he olsewhere recorder, in the collection and examination of npwards of $\mu(1),(0)$ random iligits; and was comsiderably surprised to find that, "agregriting the results, cach cligit peesented itself, with unexpected 
frequency, less than $\frac{1}{1}(0)$ of the number of times. For instance, in 100 sets of 150 digits each, I found that a digit presented itself in $n$ set more frequently under 15 times than over 15 times; similarly in the cuse of 80 sets ench of 250 digits, and also in other aggregations. Attempts to get rid of the discrepancy proved futjle, it reappearing with such persistency as to demand an explanation. Doubts arose at lirst as to the randomness of the digits; but subsequent laborions calculations of each separate term of $\left(\frac{9}{10}+\frac{1}{10}\right)^{250}$ and $\left(\frac{9}{10}+\frac{1}{1 i i}\right)^{150}$, to six places of decimals, elicited the fact that the discrepancy wiss to be expected; and, a like result persistently appearing in other numerical expansion tests, the idea was suggested of examining closely the relintion between the sum of the first $u$ terms and the sum of the last un terms in the gencial expansion of $\left(a+1+\frac{1}{a+1}\right)^{n(n+1)}$.

Strange to say, although mathematicians of the highest eminence have, ever since the discovery of the binomial theosem, devoled themselves to the scientific treatment of probability, and even in some cases to its unscientific treatiment (in such justances as the credibility of witnesses, or the fallibility of juries, or: discussions as to whether the acquisition of $£ 100$ is of greater or less "importance" or "value" to a minn possessing $£ 10,000$ than is tlie acquisition of $£ 10$ to a man possessing $£ 1000$ !), there secms no trace of previons investigations of this particular matter. It is true that, when $u$ is very large, it has been constantly assumed that the two sums of terms auc almost exactly ceural; hut even this, I helieve, has never been strictly demonstrated," nor laave attempts been minte to show to whith closeness of approximation the equality may be relied on. Moreover, as we shatl see in Art. 22, it is necessinly that not $n$ only, but $\frac{n}{a+1}$ also, should be a large number, if the two sums of terms are tot ho approximately expal at all.

3. In what follows, we shall pressume thronghont that $n, 4, l$ ane positive integers, and $a$ always greater than $b$; i.e., in no casc less

* 'Whe assumption has cromerally leen lased (no fal as T lave observed) on the fact that, when $n$ is infinite and $r$ finite, the $r$ h telm locforo the $(n b+1)^{\text {th }}$ in $\left(\frac{a}{a+b}+\frac{b}{a+b}\right)^{n+t+n}$ differe only infinitesimally from the rth term ilfere it; no aceount beiner taken, (i.) of what happene when $r$ lecomes culut, for iustance, to dinb or thercabonts, nor of the fact (ii.) that the sum of an infinite number of infinitesimal differences may itsolf represent $\because$ finite diflerence, nor (iii.) that the termss after the $(n b+1)^{\text {th }}$ infinitely exceel, in number, the terms before it. 
than 2. In the expansion of $\left(\frac{a}{a+b}+\frac{b}{a+b}\right)^{\text {natnd }}$ in descending powers of $a$, the greatest term is the $(n b+1)^{\text {th }}$, which may also be called the neutral term. 'The first $n b$ terms may conveniently be denominated tho, short-side, and the last na terms the long-side, of the expmusion.

When the odds ngainst an event are $a$ to $b$, and the number of trials is a multiple of $a+b$, we shall enll it a complete set of trials; when not a multiple of $a+b$, it may be called a broken set.

Wo will investigate the most important case first-it is

\section{Cimnplate Sets.}

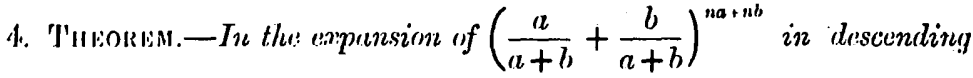

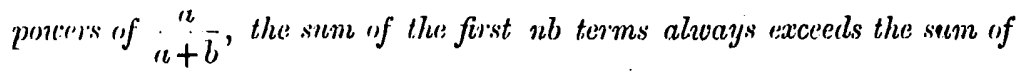
the last na terms; the excess is a maximum when $u=1$, ancl constantly diminisines as $n$ increases, lying alnvays between $\frac{1}{3} \frac{n-b}{a+b}$ times the grendest term in $\left(\frac{a}{a+b}+\frac{b}{a+b}\right)^{n a+n b}$ and $\frac{1}{3} \frac{a-b}{a+b}$ times the grearest lim in $\left(\begin{array}{c}a \\ a+b\end{array}+\frac{b}{a+b}\right)^{(n+1)(n+b)}$; its ultimate value, when $n$ is very large,

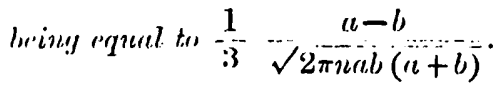

Formital:-(1) If the excoss be cleveloped in powers of $\frac{1}{n}$, its first two terms aro

$$
\frac{1}{3} \frac{n-b}{a+b}\left\{1-\frac{4}{45 n} \quad \begin{array}{cc}
(u+2 b)(2 u+b) \\
a l n(u+b)
\end{array}\right\} G_{n},
$$

where $C_{n}$ is tho grentest term for the index $n a+n b$.

(2) If the sum of the first $n h$ terms of the expansion be similarly devcloped, its first three terms are

$$
\frac{1}{2}-\frac{a+2 b}{3(a+b)}\left\{1+\frac{2}{4.5 n} \frac{(a-b)(2 n+b)}{a b(a+b)}\right\} G_{n}
$$

with is correspomling formula for the sum of the last na terms. 
5. For the sake of brevity, we will employ the symbol $(a, b)^{r}$ to denote the $r^{\text {th }}$ power of $\frac{a}{a+b}+\frac{b}{a+b}$, nnd the symbol $(a, 1)^{r}$ to denote the $r^{\text {th }}$ power of $\frac{a}{a+1}+\frac{1}{a+1}$.

Now it will easily be seen, if we multiply the reparate terms of $(a, b)^{r}$ by the separate terms of $\left.(a, b)\right)^{1}$, that the sum of the first: $p$ terms of $(a, b)^{r+1}$ is less than the sum of the first $p$ terns of $(a, b)^{r}$,

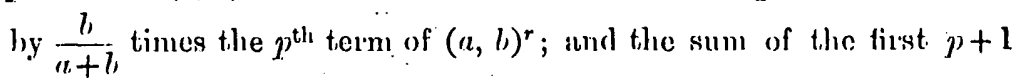
terms of $(a, b)^{r+1}$ is grenter. than the simm is the first $p$ terms of $(a, b)^{r}$, by ${ }_{n+l}^{a}$, times the $(p+1)^{\text {in }}$ term of $(a, b)^{r}$.

'I'lis heing premised, and employing $S_{n+n+t}$ to denote the shorl-

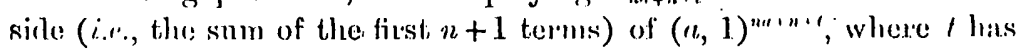
any value fiom 1 to $"+1$, both inclusive; and $S_{n+1}$ to tenotes the

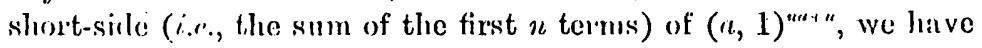

$$
(u+1)^{1, n} \text { term of }(a, 1)^{m+n}=a_{n, n}
$$

hiserefore

$$
S_{n+\cdots}-S_{n+\cdots+1}=-\frac{n}{n+\mathrm{j}} C_{n}
$$

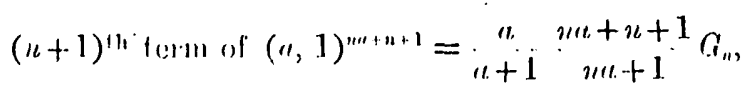

therefore $\quad S_{n+n+1}-S_{n a+n+2}=\frac{1}{n+1} \frac{n+1}{n+\frac{1}{1}} r_{n}$;

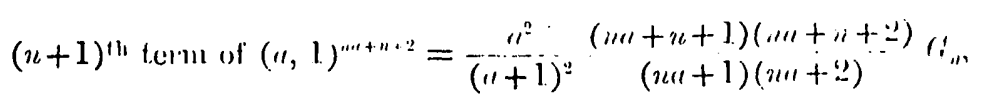

therefores $\quad S_{n+n+2}^{\prime}-S_{n+n+3}^{\prime}={ }_{n+1} \frac{\left(n+\frac{1}{n+1}\right)\left(n+\frac{2}{n+1}\right)}{\left(n+\frac{1}{n}\right)\left(n+\frac{2}{n}\right)} n_{n} ;$

and so on, till we arrive at

$$
S_{m+\ldots+\ldots, \ldots}^{\prime}-S_{(n+1,(\ldots+1) \cdot}^{\prime}
$$


Hence, by addition, $\quad S_{n(a+1)}^{\prime}-S_{(n+1)(n+1)}^{\prime}$

$=-\frac{a}{n+1} G_{n}+\frac{1}{n+1} C_{n}\left\{\frac{n+\frac{1}{a+1}}{n+\frac{1}{a}}+\frac{\left(n+\frac{1}{a+1}\right)\left(n+\frac{2}{a+1}\right)}{\left(n+\frac{1}{n}\right)\left(n+\frac{2}{a}\right)}+\ldots\right.$ to $a$ terms $\}$,

the $r^{\text {th }}$ term inside the bracket being

$$
T_{r}=\frac{\left(n+\frac{1}{a+1}\right)\left(n+\frac{2}{n+1}\right) \ldots\left(n+\frac{r}{n+1}\right)}{\left(n+\frac{1}{n}\right)\left(n+\frac{2}{a}\right) \ldots\left(n+\frac{r}{a}\right)} .
$$

We shall consequently have to find tho value of

$$
T_{1}+T_{2}+\ldots+T_{n}^{\prime} \text {. }
$$

6. I'mploying a well-known formula (see, for instance, Todhunter's Algelru, 1. 557, Jix. 155), we have

$$
T_{r}=\frac{1+\frac{r(r+1)}{2 n(a+1)}+\frac{(v-1) r(r+1)(3 r+2)}{24 u^{3}(u+1)^{2}}+\ldots}{1+\frac{r(v+1)}{2 n u}+\frac{(r-1) r(r+1)(3 r+2)}{24 n^{2} a^{2}}} .
$$

Denote this by

$$
1-\frac{n}{u}+\frac{y}{u^{2}}
$$

multiplying, and equating coeflicients, we easily obtain a: and $y$, giving

$$
\begin{aligned}
& T^{\prime},=1-\frac{1}{n} \frac{r(r+1)}{2 n(a+1)}+\frac{1}{u^{2}} \frac{r(r+1)}{24 n^{2}(a+1)^{2}}\left\{3 r^{2}+r(8 n+7)+4 a+2\right\} \\
& \text { terms in } \frac{1}{u^{3}}, \frac{1}{u^{i}}, \text { de. }
\end{aligned}
$$

Summing the values of this expression from $r=1$ to $r=a, a$ process which, though somewhat tclious, presents no difficulty, we olstain

$$
I_{1}+Y_{2}+\ldots+W_{a}=u-\frac{1}{u} \frac{u+2}{6}+\frac{1}{u^{2}} \frac{(u+2)\left(13 a^{2}+21 u+6\right)}{120 a(u+1)}+\ldots
$$

so that

$$
S_{(n+1)(n+1)}-S_{n(n+1)}
$$

$$
=\frac{a+2}{6(u+1)}\left\{\frac{1}{u}-\frac{1}{u^{2}} \frac{13 u^{2}+21 u+6}{20 u(u+1)}+\text { terms in } \underset{n^{3}}{1}, \text { \&c. }\right\} r_{n} .
$$


7. If $G_{n+1}$ denote the greatest term in $(a, 1)^{(n+1)(a+1)}$, we have

$$
\begin{aligned}
\frac{G_{n+1}}{G_{n}} & =\frac{\{n(a+1)+1\}\{n(a+1)+2\} \ldots\{n(a+1)+a+1\}}{\{n a+1\}\{n a+2\} \ldots\{n a+a\}(n+1)} \frac{a^{a}}{(a+1)^{a+1}} \\
& =\frac{\left(n+\frac{1}{a+1}\right)\left(n+\frac{2}{a+1}\right) \ldots\left(n+\frac{a}{a+1}\right)}{\left(n+\frac{1}{a}\right)\left(n+\frac{2}{a}\right) \ldots\left(n+\frac{a}{a}\right)} .
\end{aligned}
$$

Substituting $a$ for $r$ in the value of $T_{r}$ given in Art. 6, we have consequently

$$
G_{n+1}=\left\{1-\frac{1}{2 n}+\frac{1}{n^{2}} \frac{11 a^{2}+11 a+2}{24 a(a+1)}+\text { terms in } \frac{1}{n^{8}}, \& c .\right\} G_{n} .
$$

8. Let now

$$
S_{n(n+1)}=\phi(a)+\left(x+\frac{y}{n}+\frac{z}{n^{2}}+\ldots\right) G_{n}
$$

where $x, y, z$ are functions of $a$, not containing $n$. Then

$$
\begin{gathered}
S_{(n+1)(a+1)}-S_{n(n+1)} \\
=\left\{x+\frac{y}{n+1}+\frac{z}{(n+1)^{2}}+\ldots\right\}\left\{1-\frac{1}{2 n}+\frac{1}{n^{2}} \frac{11 n^{2}+11 a+2}{24 a(a+1)}+\ldots\right\} G_{n} \\
-\left\{x+\frac{y}{n}+\frac{z}{n^{2}}+\ldots\right\} G_{n}
\end{gathered}
$$

hy the preceding article. Expanding $\frac{1}{n+1}$, and equating coefficients of $\frac{1}{n}$ and $\frac{1}{n^{9}}$ in the resultant expression with the corresponding coefficients in the expression at the end of Art. 6, we easily obtain the values of $x$ and $y$, giving

$$
S_{n(a+1)}=\phi(a)-\frac{a+2}{3(a+1)}\left\{1+\frac{1}{n} \frac{2(a-1)(2 a+1)}{45 a(a+1)}+\ldots\right\} G_{n} .
$$

9. Let $\Delta_{n}$ denote the excess of the short-side over the long-side of $(a, 1)^{n a+n}$. Then, the sum of all the terms in the expansion being 
equal to unity, we have

$$
\begin{aligned}
& \Delta_{n}=2 S_{n(a+1)}-1+G_{n} \\
& =\psi(a)+\frac{a-1}{3(a+1)}\left\{1-\frac{1}{n} \frac{4(a+2)(2 a+1)}{45 a(a+1)}+\ldots\right\} G_{n}, \\
& \psi(a)=2 \phi(a)-1 .
\end{aligned}
$$

where

It seems fairly evident a priori that $\psi(a)$ must $=0$, but attempts at u rigid demonstration have given more trouble than anything else in the present paper. The unknown terms in $\frac{1}{n^{2}}, \frac{1}{n^{3}}$, \&c., preclude sny tests by numerical examples when $n$ is finite, and when $n$ is infinite numerical tests are not possible. How dlo we know but that $\psi(a)$ is some quantity which for any given value of. $a$ is infinitesimal, and not absolute zero? The difficulty, by abstract methods, lies in not "begging the question" in the very act of proving it. And, if we left it uncertain, many of the conclusions which are about to follow would be inadmissible, since our present subject consists largely in the comparison, by addition and subtraction, of other infinitesimal quantities, among which the existence of an unknown intinitesimal $\psi(a)$ would work sad havoc; and we nust not make the unnuthorized assumption which we adversely criticised in the footnote to page 291 .

It may be sufticient here, however, to assure the render that wo shall prove, by a very indirect but perfectly conclusive method in Art. 38, that $\psi(a)=0$ absolutely. Assuming it in the meanwhile, we obtain

$$
S_{n(a+1)}=\frac{1}{2}-\frac{a+2}{3(a+1)}\left\{1+\frac{1}{n} \frac{2(a-1)(2 n+1)}{45 a(a+1)}+\ldots\right\} a_{n}
$$

10. The method of A.tis. 5-8 may le utilizen to lime the sum of the short-side terms of $(a, b)^{m+n+m}$. $\Lambda \mathrm{s}$ in $\Lambda$.t. 5 , we oblain, for the cxcess of the first $n b$ terms of $(a, b)^{n a+n b}$ over the first $u b+1$ terms of $(a, b)^{(n+1)(a+b)}$, the expression

$$
-\frac{a}{a+b} G_{n}+\frac{b}{a+b}\left\{\frac{u+\frac{1}{a+b}}{n+\frac{1}{u}}+\frac{\left(n+\frac{1}{n+b}\right)\left(n+\frac{2}{n+b}\right)}{\left(n+\frac{1}{a}\right)\left(n+\frac{2}{a}\right)}+\ldots\right\} G_{n} .
$$

The series inside the bincket will have to be summed to $a+b-\mathbf{l}$ insteal of $a$ terms, leading to an expression nuch more complicated 
than the one for $T_{1}+\ldots+T_{a}$ in Art. 6 . It will also be noted that the short-side of $(a, b)^{(n+1)(a+b)}$ consists of $n b+b$ terms in all: we must therefore express the sum of the remaining $b-1$ terms in the form of the product of $G_{n}$ by some function of $a, b$, and $n$. Combining this with the former expression, the result will be obtained. There is no difficulty about the method, but the expressions become extremely' cumbrous and unwieldy. My own attempt resulted finally in a coefficient of $\frac{1}{n^{3}}$ containing 19 terms involving powers of $b$ from $b^{5}$ downwards combined with powers of $a$ from $a^{8}$ downwards. This kind of work seems needless, however, as a little consideration will, I think, in the next article suffice to show that the formula for $(a, b)^{n a+n b}$ can be immediately deduced from that for $(a, 1)^{n a+n}$.

11. The formula of Art. 9 is, of course, applicable when $h$ is aubstituted for $a$, and $n b$ for $n$, giving

$$
S_{n b(h+1)}=\frac{1}{2}-\frac{h+2}{3(h+1)}\left\{1+\frac{1}{n} \frac{2(h-1)(2 h+1)}{45 h(h+1)}+\ldots\right\} G_{n b}
$$

This expresses the sum of the first $n l$ terms of the expansion of $(h ; 1)^{n b n+n b}$ in the form of a constant, together with a function of $h$ and $n$, a factor of the latter being the $(n b+1)^{\text {th }}$ term of the expansion. Now, if $h$, instead of being an integer, were a fraction, ench of the first $n b+1$ terms of the expansion would be of the same algebraical form, and therefore we may infer that any relation connecting them, by way of addition or multiplication, would remain of the samo algebraical form also. The argument is the same as in what is called Euler's proof of the binomial theorem. Putting therefore

$$
h=\frac{a}{b}
$$

we have for the sum of the first $n b$ terms of

$$
\left(\frac{a}{b}, 1\right)^{n b \cdot\{(a l b)+n b}
$$

that is, for the short-side of the expansion of

$$
\left(\frac{a}{a+b}+\frac{b}{a+b}\right)^{n a+n b},
$$


the expression

$$
\frac{1}{2}-\frac{a+2 b}{3(a+b)}\left\{1+\frac{2}{45 n} \frac{(a-b)(2 a+b)}{a b(a+b)}+\ldots\right\} G
$$

where $G$ denotes the greatest term of the expansion.

12. We can, in fact, demonstrate the validity of the above' inference in the case of one particular fractional value of $h$. For the sum of the first $n$ terms of $(b, 1)^{n b+n}$ is, by Art. 9,

$$
\frac{1}{2}-\frac{b+2}{3(b+1)}\left\{1+\frac{1}{n} \frac{2(b-1)(2 b+1)}{45 b(b+1)}+\ldots\right\} G_{n} .
$$

Adding $G_{n}$ to this, and subtracting from 1, we have, for the sum of the remaining $n b$ terms, that is, for the sum of the first $n b$ terms of

or of

$$
\begin{gathered}
\left(\frac{1}{1+b}+\frac{b}{1+b}\right)^{n b+n} \\
\left\{\frac{\frac{1}{b}}{\frac{1}{b}+1}+\frac{1}{\frac{1}{b}+1}\right\}^{n b(1+1 / n)}
\end{gathered}
$$

in descending powers of $\frac{\frac{1}{b}}{\frac{1}{b}+1}$, the expression

$$
\begin{gathered}
\frac{1}{2}-\frac{1+2 b}{3(b+1)}\left\{1-\frac{1}{n} \frac{2(b-1)(2+b)}{45 b(b+1)}+\ldots\right\} G_{n} \\
\text { or } \quad \frac{1}{2}-\frac{\frac{1}{b}+2}{3\left(\frac{1}{b}+1\right)}\left\{1+\frac{1}{n} \frac{2\left(\frac{1}{b}-1\right)\left(\frac{2}{b}+1\right)}{45 \frac{1}{b}\left(\frac{1}{b}+1\right)}+\ldots\right\} G_{n}
\end{gathered}
$$

which proves the validity of substituting $\frac{1}{b}$ for $h$ in the formula of Art. 11.

13. Hence, if $\Delta_{n}$ denote the excess of the short-side over the longside of $(a, b)^{n n+n b}$, we have, as in Art. 9,

$$
\Delta_{n}=\frac{a-b}{3(a+b)}\left\{1-\frac{1}{n} \frac{4(a+2 b)(2 a+b)}{45 a b(a+b)}+\ldots\right\} G_{n},
$$


$G_{n}$ being the greatest term of the expansion. Moreover, if $G_{n+1}$ be. the greatest term in $(a, b)^{(n+1)(a+b)}$, we have, as in Art. 7,

$$
G_{n+1}=\left\{1-\frac{1}{2 n b}+\ldots\right\} G_{n} .
$$

Now $\frac{1}{2 b}$ is, for all integral values of $a$ and $b$, where $a>b$, greater than $\frac{4(a+2 b)(2 a+b)}{45 a b(a+b)}$. Therefore $\Delta_{n}$ lies between $\frac{1}{3} \frac{a-b}{a+b} G_{n}$ and $\frac{1}{3} \frac{a-b}{a+b} G_{n+1}$; and, subject to a reservation, the theorem of Art. 4 is: proved.

14. The reservation is this: that the terms involving $\frac{1}{x^{3}}, \frac{1}{x^{4}}, \& c .$, which are infinite in number, although of no importance when $n$ is large, may, for anything we know at present, form such seriously disturbing elements when $n$ is small as to render the above formulio for such a case practically useless. And each coefficient being some unknown function of $a$ and $b$, one would imagine that such disturbance would increase when the ratio of $a$ to $b$ became larger. No one would anticipate a priori that, when $n=1$, and $a=\infty$, for instance, the formula of Art. 9 would be of any value whatever.

But it will be found on trial that, even for the severe test-case of $n=1$, the formula does, in a surprising manner, give a very fairly close approximation to the actual value of $\Delta_{n}$, obtained by independent calculation. With a view to demonstrate this, I have prepared a table, whose second column gives, for assigned values of $a$, thevalue of

$$
\frac{1}{3} \frac{a-1}{a+1} G_{n}
$$

the third column the value of

$$
\frac{1}{3} \frac{a-1}{a+1}\left\{1-\frac{1}{n} \frac{4(2 a+1)(a+2)}{45 a(a+1)}\right\} G_{m}
$$

the fourth column the independently obtained value of $\Delta_{n}$, and the linst column the ratio of the "error" to the exuct value of $\Delta_{n}$. Great cirire has been taken in the calculations, but, as I have been unable to get them tested by an independent mind, inaccuracies may here and there have crept in. These would not, however, if existent, affect the general conclusions deducible from the table as a whole. 
15. From $a=4$ to $a=8$, the calculation is for $(a+1)^{n a+n}$; the "proportion of error" is, however, the same as if the denominator's vere included.

$$
n=1, \quad b=1 \text {. }
$$

\begin{tabular}{|c|c|c|c|c|}
\hline & $\begin{array}{c}\text { First } \\
\text { Approximation. }\end{array}$ & $\begin{array}{c}\text { Second } \\
\text { Approximation. }\end{array}$ & $\begin{array}{l}\text { Actual } \\
\text { Value. }\end{array}$ & $\begin{array}{l}\text { Proportion } \\
\text { of Error. }\end{array}$ \\
\hline$a=2$ & $\cdot 049383$ & .034751 & .037037 & $\frac{1}{16}$ \\
\hline$a=3$ & $\cdot 070313$ & .052084 & .054687 & \\
\hline$a=4$ & 256 & $194 \frac{1}{2} \frac{6}{3}$ & 203 & 4 \\
\hline$a=5$ & $4166 \frac{3}{3}$ & $3216_{\frac{4}{81}}$ & 3344 & $\frac{1}{6}$ \\
\hline$a=6$ & 77760 & 60645 & 62921 & \\
\hline$a=7$ & 1647086 & 1294139 & $13407: 30$ & $\frac{1}{2} \cdot \overline{8}$ \\
\hline$a=8$ & $39146837 \frac{1}{3}$ & 30940834 & 32009911 & $\frac{1}{2 \theta \cdot \theta}$ \\
\hline$a=9$ & $\cdot 103312$ & .081986 & .084777 & $\frac{1}{30 \cdot 3}$ \\
\hline$a=24$ & $\cdot 1151261$ & 0933978 & .0962064 & $\frac{1}{3 \frac{1}{4} 2}$ \\
\hline$u=100$ & $\cdot 120797$ & .099002 & $\cdot 101813$ & $\frac{1}{36 \cdot 2}$ \\
\hline$a=1000$ & $\cdot 122443$ & $\cdot 100642$ & $\cdot 103454$ & $\frac{1}{30 \cdot 79}$ \\
\hline$a=\infty$ & $\cdot 12262648$ & $\cdot 10082622$ & $\cdot 103638: 32$ & $\frac{1}{36 \cdot 856}$ \\
\hline
\end{tabular}

The above results show, I venture to think, beyond all question, that the formula-value never differs from the actual value by more than $\frac{1}{30}$, and, when $a$ is greater than 8 , never by more than $\frac{1}{30}$; and that, whenever $u$ is not less than 100, it gives almost exactly $\frac{8}{3} \frac{\pi}{6}$ or $\frac{3}{3} \frac{\pi}{7}$ of the actual value. For the extremely severe test-case of $n=1$, this approximation is closer than the most sanguine investigator would have dared to liope beforehand.

16. The following are other miscellaneous test-cases, calculated with all possible care; in some instances it has been necessary to use ten-figure lognrithms; and many of the calculations in the fourth column are extremely lahorious. 
$b=1$.

\begin{tabular}{|c|c|c|c|c|c|}
\hline & & $\underset{\text { Approximation. }}{\text { First }}$ & $\begin{array}{c}\text { Second } \\
\text { Approximation. }\end{array}$ & $\begin{array}{l}\text { Actual } \\
\text { Value. }\end{array}$ & $\begin{array}{l}\text { Proportion } \\
\text { of Error. }\end{array}$ \\
\hline$a=2$, & $n=2$ & .036580 & .031164 & 031550 & $\frac{1}{82}$ \\
\hline$"$ & $n=3$ & .030348 & .027351 & .027485 & $\frac{1}{205}$ \\
\hline$"$ & $n=4$ & .0264940 & .02453148 & .02459540 & $\frac{1}{84}$ \\
\hline$"$ & $n=5$ & $\cdot 02381189$ & 02240082 & .02243662 & $\frac{1}{6} \overline{7}$ \\
\hline$"$ & $x=6$ & .02180755 & .0207306 & .0207529 & $-\frac{1}{30}$ \\
\hline$"$ & $u=7$ & .0202365 & .0193799 & 0193949 & $\frac{1}{1200}$ \\
\hline$"$ & $n=8$ & $\cdot 0189624$ & 0182601 & 0182706 & $\frac{1}{1730}$ \\
\hline$a=3$ & $x=2$ & .0519100 & .0451800 & .0456240 & $\frac{1}{105}$ \\
\hline$"$ & $n=3$ & $\cdot 0430100$ & 0392997 & 0394536 & $\frac{1}{258}$ \\
\hline$"$ & $n=4$ & .0375332 & .0351012 & .03517326 & $\frac{1}{488}$ \\
\hline " & $n=5$ & .03372185 & 03197331 & .03201413 & $\frac{1}{784}$ \\
\hline$a=9$ & $n=2$ & .076047942 & .068199043 & .068673713 & $\frac{1}{145}$ \\
\hline$a=99$, & $n=2$ & 0888641163 & $\cdot 080845800$ & .081324387 & $\frac{1}{169}$ \\
\hline$a=\infty$, & $n=2$ & .090223523 & .082203654 & .082682266 & $\frac{1}{73}$ \\
\hline
\end{tabular}

The foregoing table exhibits a regularity which cannot be the result of accident, and which leads to the sure conviction that the $\Delta_{n}$ formula, which we have strictly demonstrated merely for large values of $n$, holds not only for the foregoing cases, but for other hitherto untested cases where $n$ is of moderate magnitude. We may, 1 think, confidently assert that, when $n=2$ and $a>2$, the "error" in the formula is never greater than $\frac{1}{10}$; when $n=3$, it is never greater than $\frac{1}{20} 0$; when $x=4$ and $a>3$, it is never greater than $\frac{1}{0} \frac{1}{0}$; when $u=5$ and $a>3$, it is never greater than $\frac{1}{8} \delta$; when $n=6$ and $a>2$, it is never greater than $\frac{1}{100 \sigma}$; and so on, the approximation of course always becoming closer as $n$ increases. It will be observed how much closer the second approximation is than the first. 
17. In order that there may be as little doubt as possible of the applicability of the formula to small values of $n$, I add yet three more tables, calculated (i.) for $a=10$, (ii.) for $a=\infty$, and (iii.) for miscellaneous values, chosen at haphazard, of $a$ and $b$.

(i.) $b=1, \quad a=10$.

\begin{tabular}{|c|c|c|c|c|}
\hline & $\begin{array}{c}\text { First } \\
\text { Approximation. }\end{array}$ & $\begin{array}{c}\text { Second } \\
\text { Approximation. }\end{array}$ & $\begin{array}{c}\text { Actunl } \\
\text { Value. }\end{array}$ & $\begin{array}{c}\text { Proportion } \\
\text { of Error. }\end{array}$ \\
\hline$n=1$ & $300000 \ldots$ & $238909 \ldots$ & $24688 \ldots$ & $\frac{1}{31}$ \\
$n=2$ & $630000 \ldots$ & $565855 \ldots$ & $56973 \ldots$ & $\frac{1}{1+\frac{1}{7}}$ \\
$n=3$ & $14880 \ldots$ & $13870 \ldots$ & $13909 \ldots$ & $\frac{1}{3 \frac{1}{5}}$ \\
$n=4$ & $370230 \ldots$ & $351382 \ldots$ & $351902 \ldots$ & $\frac{1}{676}$ \\
$n=5$ & $9487530 \ldots$ & $9101129 \ldots$ & $9109467 \ldots$ & $\frac{1}{104 \overline{1}}$ \\
$n=6$ & $24779664 \ldots$ & $23938657 \ldots$ & $2395353 \ldots$ & $\frac{1}{16 \overline{1}}$ \\
\hline
\end{tabular}

The above calculation is for $(a+1)^{n(a+1)}$, without the denominators, and only the first few figures of eacl number are given. Sorne of the latter calculations necessitate the finding of $\log 11$ to at least ten places of decimals.

(ii.) $b=1, a=\infty$.

\begin{tabular}{|c|c|c|c|c|}
\hline & $\underset{\text { Approximation. }}{\text { First }}$ & $\begin{array}{c}\text { Second } \\
\text { Approximation }\end{array}$ & $\begin{array}{l}\text { Actual } \\
\text { Value. }\end{array}$ & $\begin{array}{l}\text { Propourtion } \\
\text { of Eerror. }\end{array}$ \\
\hline$n=1$ & $\cdot 12262648$ & $\cdot 10082622$ & $\cdot 10363832$ & $\overline{3} \overline{6} \cdot \frac{1}{56}$ \\
\hline$n=2$ & .090223523 & .082203654 & $\cdot 082682266$ & $T_{17}^{\frac{1}{7} \overline{3}}$ \\
\hline$n=3$ & .074680603 & .070255085 & .070421970 & $\frac{1}{4} \frac{1}{2}$ \\
\hline$n=4$ & $\cdot 065122268$ & $\cdot 062227945$ & .062307055 & $\frac{1}{8} \overline{7}$ \\
\hline$x=5$ & $\cdot 058489123$ & $\cdot 056409510$ & $\cdot 056453940$ & $\frac{1}{1270}$ \\
\hline$u=6$ & $\cdot 053541047$ & $\cdot 051954646$ & $\cdot 05198242 ; 3$ & $\frac{1}{18} \frac{1}{7} 1 \sqrt{3}$ \\
\hline
\end{tabular}


In calculating the above table, I have utilized the values of $e^{-p}$ piven in Part II. of Mathematical Tracts, by Professor F. W. Newman.

(iii.)

\begin{tabular}{|c|c|c|c|c|}
\hline & $\underset{\text { Approximation. }}{\text { First }}$ & $\begin{array}{c}\text { Second } \\
\text { Approximation. }\end{array}$ & $\begin{array}{l}\text { Actual } \\
\text { Value. }\end{array}$ & $\begin{array}{l}\text { Proportion } \\
\text { of Error. }\end{array}$ \\
\hline$(3+2)^{5}$ & 72 & $60 \frac{4}{75}$ & 61 & $\frac{1}{64}$ \\
\hline$(3+2)^{10}$ & 163296 & 149749 & 150223 & $\frac{1}{300}$ \\
\hline$(5+2)^{7}$ & 37500 & $32357 \cdot \frac{1}{7}$ & 32707 & $\frac{1}{9}$ \\
\hline$(9+2)^{11}$ & $180796 \ldots$ & $159694 \ldots$ & $160820 \ldots$ & $1 \frac{1}{4} \overline{3}$ \\
\hline$(9+2)^{23}$ & $372635 \ldots$ & $350888 \ldots$ & $351508 \ldots$ & $\frac{1}{567}$ \\
\hline$(10+3)^{20}$ & $301247 \ldots$ & $288614 \ldots$ & $288844 \ldots$ & $\frac{1}{1250}$ \\
\hline
\end{tabular}

In the above, the denominator's of $a$ and $b$ have, for the sake of simplicity, been onitted; and in the last thice cases, the numbers leing very large, only their first few digits are given.

18. It is now, I think, abundantly manifest that the $\Delta_{n}$ formula may be confidently applied, within limits such as those indicated in $\Lambda \mathrm{rts}$. 15 and 16, to small as well as large values of $\pi$. It is carious to note that the $G_{n+1}$ formula in terms of $G_{n}$ (see Art. 7), from which the $\Delta_{n}$ formula is partly derived, dues not, for small values of $u$, givo approximations nearly so close to the actual values as does the $\Delta_{n}$ formula itself. For instance, when $\iota=\infty, b=1, u=2$, we obtain

First Approximation. Sccond Approximation. Actuul Value of $G_{n+1}$. $\cdot 203003$ $\cdot 234017$ .224042

so that the second approximation (to three terms) differ's by more than $\frac{-1}{2} \overline{\mathrm{s}}$ from the actual value, a much wider deviation than for the coryesponding case of $\Delta_{n}$ (sec Art. 16), where the "jpoportion of error" is only $\frac{1}{1} \overline{7}$.

19. The closeness of the approximation of the $\Delta_{n}$ formula, and the fact of its becoming closer as $a$ increases, may enable us to surmise 
the nature of the terms that follow. Since $\Delta_{n}$ clearly vanishes altogether when $a=b$, it is fairly evident that $a-b$ must be a factor of each separate term. Again, the interchange of $a$ and $b$ will give the excess of the long-side over the short-side, which is $-\Delta_{n}$; therefore the remaining factors of each term must form a symmetric function of $a$ and $b$. A little consideration will, moreover, show that the substitution of $\frac{1}{a}$ for $a$, and $\frac{1}{b}$ - for $b$, and $n a b$ for $n$, will also give the excess of long-side over short-side, or $-\Delta_{n}$ (compare Art. 12). 'Therefore the above substitution in any term must lead to the same result as the substitution of $b$ for $a$, and $a$ for $b$. This would seem to imply that the coefficient of $\frac{1}{u^{p}}$ is of the form

$$
\frac{(a-b) \phi(a, b)}{a^{\nu} b^{p} \psi(a, b)}
$$

where $\phi(a, b)$ is a symmetric homogeneous function of $a$ and $b$, of $p-1$ dimensions higher than the other symnetric lomogreneous function $\psi(a, b)$. Again, the substitution of $\frac{a}{a+b}$ for $a, \frac{b}{a+b}$ for $b$, and $n(a+b)$ for $u$, would make no alteration in any term of the original expansion, and therefore could make no alteration in the formula for $\Delta_{u}$; whence it would follow that $(a+b)^{p}$ is a factor of $\psi(a, b)$. Once more, the denominator of the coefficient of $\frac{1}{x^{\prime \prime}}$ in the formula for $~ T$.'. in Art. 6 will be found, on consideration, to contain $a^{p}(a+1)^{p}$ as $a$ fuctor, and no other factor involving $a$; nor can such a factor be introduced by the summation, fiom $r=1$ to $r=a$, of any rational integral function of $r$. Observing the formula of Art. 7, we see therefore that the denominator of the coefficient of $\frac{1}{x^{p}}$ in the $\Delta_{n}$ formula of Art. 9 cannot contrin a function of $a$ of higher dimensions than $a^{p}(a+1)^{p+1}$. Combining this conclusion with those obtained above, it would seem clear that the coefficient of $\frac{1}{u^{\nu}}$ in the $\Delta_{n}$ for'mula of Art. 13 must be of the form

$$
\frac{(a-b) \phi(a, b)}{k a^{\prime \prime} b^{\prime \prime}(a+b)^{\prime+1}}
$$

where $k$ is some integer; and $\phi(a, b)$ is a symmetric homogeneous 
function of $a$ and $b$ of $2 p$ dimensions. We may therefore suppose that

$$
\begin{aligned}
\Delta_{n}=\frac{a-b}{3(a+b)}\left\{1-\frac{1}{n} \frac{8 a^{2}+\ldots}{45 a b(a+b)}\right. & +\frac{1}{n^{2}} \frac{l_{9} a^{4}+\ldots}{k_{8} a^{2} b^{3}(a+b)^{2}} \\
& \left. \pm \frac{1}{n^{8}} \frac{l_{8} a^{0}+\ldots}{k_{8} a^{8} b^{8}(a+b)^{8}}+\ldots\right\} G_{m},
\end{aligned}
$$

where the omitted terms contain the residue of symmetric homogeneous functions of $a$ and $b$.

Now, if the quantities $1,-\frac{8}{45}, \frac{l_{2}}{k_{2}}, \frac{l_{3}}{k_{3}}, \frac{l_{4}}{k_{4}} \ldots$ form a converging series, we shall at once account for the fuct that the first two terms give a very close approximation to the value of the whole series, even when $u=1$. It would also explain why the approximation is closer for large than for small values of $a$ (in comparison with $b$ ), if wo werc to suppose that the coefficients of omitted terms in the residue of the numerators would lead to series converging less rapidly.

I have no doubt that the above hypothesis (which we shall have an opportunity of testing in Art. 40) is correct; and no doubt also that the coefficients of the $G_{n+1}$ formula are subject to the same law, but that they give rise to a series which converyes less rapidly.

20. By observation of the table of Art. 16, and the second table of Art. 17 (the first table of Art. 17 being for this purpose clearly inapplicable), it will be seen how the "actual value" of $\Delta_{r}$ in those cases always lies about midway between the "first approximations" for $n=r$ and $n=r+1$. In several other instances where $b=1, \mathrm{I}$ have also tested that, for small values of $n, \Delta_{n}$ always lies about nidway between $\frac{1}{3} \frac{a-b}{a+b} G_{n}$ and $\frac{1}{3} \frac{a-b}{a+b} G_{n+1}$; the matter seems sufficiently clear without being pursued any further.

The reservation made at the end of Art. 13 may now be removed, and I trust that I may be allowed to assert confidently that the theorem of Art. 4 is completely proved; and that the formula there given may be unhesitatingly applied, within close approximations, for small as well as for large values of $n$.

When $n$ is very large, the term involving $\frac{1}{n}$ may be omitted; and we then obtain, by a well-known formula for $G_{n}$,

vOL. XXYI.-NO. 519.

$$
\Delta_{n}=-\frac{1}{3} \frac{a-b}{\sqrt{2 \pi n a b(a+b)}} \text {. }
$$


21. The applicability of the theorem to the scientific theory of gambling is manifest. It proves that, at the end of any complete set of $n(a+1)$ trials, a man who gives odds of $a$ to 1 in a fair wager is more likely to have made a net gain than a net loss. When $n$ is very large, the excess of his whole chance of gaining over his whole chance of losing is, of course, extremely minute; but that it should be always measurable and always positive is fairly subversive of hitherto accepted notions.

But is the largeness of $n$ the only condition necessary for the minuteness of the excess? Let us see. Suppose $a=99, n=10$. The formula (corrected in accordance with Art. 16) gives $\Delta_{n}=\cdot 04068$, whence short-side $=\cdot 4570$, long-side $=4162$, the neutral term $G_{n}$ being 1268 . Here the difference between 45 and 41 is very considerable; showing, as it does, that a man who bets 99 to $I$ in a fair wager is, after 1000 trials, more likely, by so much as 10 per cent., to have made a net gain than a net loss. Odds of 99 to 1 may be considered rather large; but insurance companies, in the course of daily business, are constantly having to deal with odds far greater.

Let us take another instance. My house is insured for a premium at the rate of $1 s .6 d$. per $£ 100$, which, translated into probability language, means that the insurance company bets me about 1333 to 1 that my loouse will not be burnt down within the next 12 months. But the company have to allow for office expenses and profits, so that the real odds, in their opinion, are greater than this; let us suppose 1499 to 1 , implying an event the probability of whose occulrence is $\frac{1}{1} \sigma_{0}^{2}$. Now 12,000 may, in probability language, be fairly considered a "very large number"; and if, six months ago, any one had asked me what was likely to happen to a millionaire who kept on making bets of 1499 shillings to 1 shilling against such an event, I should have confidently replied that after 12,000 trials he was just as likely to have made a net loss as a net gain. Well, let us put $a=1499, b=1, n=6$ in the formula. We obtain

$$
\Delta_{n}=06136, \quad G_{n}=\cdot 18816 ;
$$

whence probability of net gain $=43660$, probability of net loss $=37524$, or probability of net gain exceeds, by so much as 17 per. cent., the probability of net loss.

22. The preceding considerations lead to an important conclusion. It is this : that, in order to secure the approximate balancing of gains and losses, it is not only necessary that the number of trials should be a 
large number, but that the product of the number of trials by the probability of the event should also be a large number. This, once pointed out, may be seen to be true on other grounds; but, strange to say, I am unable to find it mentioned by any previous writer.

On the contrary, a writer of deservedly high repute tells us: "If the probability of an event be $p$, then, out of $N$ cases in which it is in question, it will happen $p N$ times, $N$ being any very large number."* The necessary condition, of course, ought to be, not that $N$ is a very large number, but that $p N$ is a very large number. A similar objection applies to the statement of another writer, to whom all students of probability are greatly indebted, viz.: "The value of a given chance of obtaining a given sum of money is the chance multiplied by that sum; for in a great number of trials this would give the sum actually realized." $\dagger$

23. We must now pass on to another portion of our subject. The examination of broken-period sets of trials will be found to lead to conclusions quite as interesting as, and still more curious than, those we have already discussed.

For want of a better term, let us use the word "advantage" to denote, after a certain number of events, in the case of a gambler who gives odds, the excess of the probability that he has made a net gain over the probability that he has made a net loss. We will make a further remark on the word in Art. 41. The symbol $A_{r}$ may conveniently be employed to denote the "advantage" after $r$ events.

\section{Broken-period Sets.}

24. Consider first the expansion of $(a, b)^{n a+n b+1}$. From Art. 5, we have

$$
S_{n a+n+1}-S_{n n+n}=\frac{a}{a+b} G_{n}
$$

and, in like manner, $\quad L_{n a+n+1}-L_{n n+n}=\frac{b}{a+b} G_{n}$,

Therefore, by subtraction,

$$
A_{n a+n+1}=A_{n a+n}+\frac{a-b}{a+b} G_{n}
$$

* Professor Chrystal, Algelira, Vol. Ir., Chap. xxxvi., \$1.

+ Professor Crofton, Article "Probability" in the Encyclopadia Britannica. The italice are my own. I have been most unwilling to cite particular authore, for almost every writer on probubility has made statements more or less equivalent; a few months ago I should certininly have dono so myself.

$$
\mathbf{x} 2
$$


showing that the "advantage" after $n a+n+1$ trials exceeds that after $n a+n$ trials by $\frac{a-b}{a+b} G_{n}$.

25. Take next the expansion of $(a, b)^{n a+n b-1}$. Here, observing that $G_{n}$ the $(n b+1)^{\text {th }}$ term of $(a, b)^{n n+n b}$ is also the $n b^{\text {th }}$, and likewise the $(n b+1)^{\text {th }}$ term of $(a, b)^{n a+n b-1}$, we have

$$
S_{n a+n b}-S_{n a+n b-1}=-\frac{b}{a+b} G_{n}
$$

and, similarly, $\quad L_{n a+n b}-L_{n a+n b-1}=-\frac{a}{a+b} G_{n}$.

Whence, by subtraction,

$$
A_{n a+n b}=A_{n n+n b-1}+\frac{a-b}{a+b} G_{m}
$$

that is to say, the "advantage" after $n a+n b-1$ trials falls short of the "advantage" after $n a+n b$ trials by $\frac{a-b}{a+b} G_{n}$; therefore, according to the formula of Art. 13, it is a negative quantity. Hence, if a broken-period set consist of one trial less than a complete set, the man who takes odds; and not the man who gives odds, has the so-called "advantage." This, however, in no way weakens the general conclusions already deduced from Art. 4, as we proceed to show.

26. The algebrnical sum of the two " advantages" after $n a+n b+1$ and $n a+n b-1$ trials is $2 \Delta_{n}$, which is positive; thus the " ndvantage" in the former caso exceeds the " disadvantage" in the latter. And if a gambler makes $n a+n b-1$, or $n a+n b$, or $n a+n b+1$ trials, all equally likely, his probable " ndvantrge," i.e., the excess of his gainchance over his loss-chance (but not the ratio of the chances), will be the same as if he were to make $n a+n b$ trials for certain. The matter is sufficiently interesting to allow of illustration by numerical examples, calculated independently of our formulr.

$$
\begin{array}{rlrl}
\left(\frac{2}{3}+\frac{1}{3}\right)^{\circ} \text { "Advantage" } & =-\frac{19}{243}, \\
\left(\frac{2}{3}+\frac{1}{3}\right)^{\circ} & \Rightarrow & =+\frac{23}{729}, \\
\left(\frac{2}{3}+\frac{1}{3}\right)^{7} & " & =+\frac{103}{729},
\end{array}
$$




$$
\begin{array}{rlrl}
\left(\frac{5}{6}+\frac{1}{6}\right)^{6} \text { "Advantage" } & =-\frac{763}{3888}, \\
\left(\frac{5}{6}+\frac{1}{6}\right)^{0} & " & =+\frac{1672}{23328}, \\
\left(\frac{5}{6}+\frac{1}{6}\right)^{7} & \quad & =+\frac{7922}{23328} .
\end{array}
$$

Note that, in each group, the middle "advantage" is the algebraical mean of the first and last.

From (ii.) we may deduce that if 139968 persons throw dice, each betting always (with some person or persons outside) 5 to 1 against the ace, and that if 46656 stop after 5 throws, 46656 after 6 throws, and 46656 after 7 throws, then we may "expect," in the aggregrate, 65625 to make a net gain, 55593 a net loss, and 18750 to end as they began. If, on the contrary, the whole 139968 had thrown 6 times and no more, we must have "expected" 4.6875 to make a net gain, $3684: 3$ a net loss, and 56250 to end as they began. The difference, but not the ratio, between the gainers and the losers is the same in both cases.

27. Putting $b=1$, we will now consider broken-period sets represented by $n(a+1) \pm p$, where $p$ lies between 1 and $a+1$. It is very easy, but superfluous, to show that the algebraical sum of the "advantages" after $n(a+1)+2$ and after $n(a+1)-2$ trials is greater than twice $\Delta_{n}$. The case is included in the proposition we are about to prove, viz., that the sum of the "advantages" after $n(a+1)+p$ and after $n(a+1)-p$ trials is greater than the like sum after $n(a+1)+(p-1)$ and after $n(a+1)-(p-1)$ trials. It may be remarked that the "advantage," for any given value of $n$, is always greatest when $p=1$, and goes on diminishing as $p$ increases, becoming negative somewhere in the latter half of the period, as $p$ approaches the value $a$.

28. Denote by $X$ the $n^{\text {th }}$ term of

$$
(a, 1)^{n a+n-p},
$$

which is $\frac{(n a+n-p)(n a+n-p-1) \ldots(n a-p+2)}{(n-1) !} \frac{a^{n a-p-1}}{(a+1)^{n a+n-p}}$.

Then we have $\quad S_{n a+n-p+1}-S_{n a+n-p}=-\frac{X}{a+1}$,

and, similarly, $\quad L_{n a+n-p+1}-L_{n n+n-p}=\frac{X}{a+1}$. 
Therefore, by subtraction,

$$
\dot{A}_{n a+n-p+1}-A_{n a+n-p}=-\frac{2 X}{a+1} .
$$

Again, denote by $Y$ the $(n+1)^{\text {th }}$ term of

$$
(a, 1)^{n a+n+p-1}
$$

which is $\frac{(n a+n+p-1)(n a+n+p-2) \ldots(n a+p)}{n !} \frac{a^{n a+p-1}}{(a+1)^{n a+n+p-1}}$.

Then, as before, $\quad A_{n a+n+p}-A_{n a+n+p-1}=-\frac{2 Y}{a+1}$.

We require therefore to prove that $X$ is greater than $Y$, which is the same thing as proving the coefficient of $z^{n-1}$ in $\left(\frac{a}{a+1}+\frac{z}{a+1}\right)^{n a+n-1,}$ greater than the coefficient of $z^{n}$ in $\left(\frac{a}{a+1}+\frac{z}{a+1}\right)^{n a+n+p-1}$.

Let $O_{n}, O_{n-1}, C_{n-2}$ denote the respective coefficients of $z^{n}, z^{n-1}, z^{n-2}$ in $(a+z)^{n+n-p}$. Then we have to show that $O_{n-1}$ is greater than the coefficient of $z^{n}$ in

$$
\frac{(a+z)^{2 p-1}}{(a+1)^{2 p-1}}\left\{\ldots+C_{n-2} z^{n-2}+C_{n-1} z^{n-1}+O_{n} z^{n}+\ldots\right\},
$$

which, since

$$
C_{n}=\frac{n a-p+1}{n a} O_{n-1}, \quad O_{n-2}=\frac{n a-a}{n a-p+2} O_{n-1}, \quad \& c .,
$$

is the same as showing that $(a+1)^{2 p-1}$ is greater than the coefficient of $z^{n}$ in

$$
\begin{aligned}
(a+z)^{2 p-1}\left\{\ldots+\frac{(n a-a)(n a-2 a)}{(n a-p+2)(n a-p+3)} z^{n-3}\right. & +\frac{n a-a}{n a-p+2} z^{n-2}+z^{n-1} \\
& \left.+\frac{n a-p+1}{n a} z^{n}+\ldots\right\},
\end{aligned}
$$

that is to say, $(a+1)^{2 p-1}>a^{2 p-1} \frac{n a-p+1}{n a}$

$$
\begin{aligned}
& +(2 p-1) a^{2 p-2}+\left(\begin{array}{c}
2 p-1 \\
2
\end{array}\right) a^{2 p-3} \frac{n a-a}{n a-p+2} \\
& +\left(\begin{array}{c}
2 p-1 \\
3
\end{array}\right) a^{2 p-4} \frac{(n a-a)(n a-2 a)}{(n a-p+2)(n a-p+3)}+\ldots
\end{aligned}
$$


where the symbol $\left(\begin{array}{l}l \\ r\end{array}\right)$ denotes $\frac{l !}{r !(l-r) !} \cdot$ Expanding $(a+1)^{2 p-1}$, and comparing the two sides term by term, it is obvious that the required inequality obtains, so long as $n a-p+2$ is not less than $n a-a$; that is, so long as $p$ is not greater than $a+2$.

Thus we have proved $A_{n n+n+2}+A_{n a+n-2}>A_{n a+n+1}+A_{n a+n-1}$, i.c., $>2 \Delta_{n}$. Similarly, $A_{n a+n+3}+A_{n a+n-3}$ is still further $>2 \Delta_{n}$, and so on, till we arrive at $A_{n n+n+(a+2)}+A_{n n+n-(n+2)}$. Our present object is, however, attained when we arrive at $A_{n a+n+p}+A_{n a+n-p,}$, where $p$ is, as nearly as possible, equal to $\frac{1}{2}(a+1)$.

29. Let us again take a particular example. Suppose a number of persons each to bet 5 to 1 against the ace, and two of them to stop when the die has been thrown once, two when it has been thrown twice, two at three times, two at four times, and so on, until the number of persons is infinite. Divide them into. equal groups: let group I. consist of one person who stops at 3 throws, one who stops at 9 , and all who stop at intermediate numbers; group II. of the other who stops at 9 , one who stops at 15 , and all who stop at intermediate numbers, and so on. The $n^{\text {th }}$ group will contain 12 persons, ranged equally on both sides of the two persons who stop at $6 n$ throws.

By the preceding article, the sum of the "advantages" of these 12 persons (i.e., the excess of the sum of their 12 chances of net gain over the sum of their 12 chances of net loss) is greater than $12 \Delta_{n}$, or than $\frac{8}{3}\left(1-\frac{154}{675 n}\right) G_{n}$. Two persons only have a chance of ending as they began, and the sum of the chances that this will happen is $2 G_{n}$. Subtracting this, we see that the excess of the sum of the 12 chances of net gain over the 12 chances of not making a net gain is $\left(\frac{2}{3}-\frac{1232}{2025 n}\right) G_{n}$. Therefore, if a person in the group be chosen at random, the excess of his chance of gaining over his chance of not gaining is $\frac{1}{12}\left(\frac{2}{3}-\frac{1232}{2025 n}\right) G_{n}$, which for all values of $n$ is positive; therefore he is more likely than not to make a net gain. The same result will ensue, whichever group we choose, also for the 5 persons we omitted before the groups began. Therefore, any person chosen at random out of the whole infinite multitude is more likely than not to have made a net gain. This, in other words, is the proposition we undertook in Art. 1 to prove, i.e., that if a die be thrown any number of 
times chosen at random, the number of appearances of the ace is more likely than not to be less than $\frac{1}{6}$ of the number of throws. The conclusion will porliaps be as surprising to the reader as it was, in the first instance, to the present writer.

30. Let us investigate the general case which includes the preceding article. The sum of the "advantages" in any complete group of $a+1$ sets of trials, whose centre is a set of $n(a+1)$ trials, is, by Art. 28 , greatcr than $(a+1) \Delta_{n}$,

i.c., greater than $\frac{1}{3}(a-1)\left\{1-\frac{1}{n} \frac{4(2 a+1)(a+2)}{45 a(a+1)}\right\} G_{n}$,

i.c., $\quad \quad \quad \quad G_{n}+\frac{1}{3}(a-4) G_{n}-\frac{1}{n} \frac{4(a-1)(2 a+1)(a+2)}{135 a(a+1)} G_{n}$,

which, whonever $a>4$, is easily seen to be $>G_{n}$.

Thus, by the reasoning of the previous article, if a person bets more than 4: to 1 in a fair wagcr, and is undecided as to how many trials he has marle, or will make, he is more likely than not finally to make a net gain. This surcly is the case of the ordinary persistent gambler who gives odds, provided that his stakes are always the same, and small compared with his means: for it may be assumed that no gambler ever kept a systematic record, perfectly accurate from boy hood, of the exact mumber of transactions he had entered upon.

31. It will be observed that in Arts. 27-30 we have assumed that $b=1$. I made many persistent attempts, by the method of Art. 11, and otherwise, occupying more time than I should like to confess, to extend the proposition of Art. 28 to the general case of $(a, b)^{n a+n b+p}$, with the result of cliscovering at the end that it is not true except when $b=1$. The method of Art. 28 for forming the successive "alvantage" -differences is not valid when $b>1$, since the number of terms in the short-side increases gradually in a most irregular and confusing way from $n b$ to $n b+b$, as we proceed from expansion to expansion between $(a, b)^{n a+n b}$ and $(a, b)^{n+n b+a+b}$. The demonstration of Art. 28, in fact, though apparently valid until $p=a+2$, will be found, on curcful examination, to be valid, even for the case of $(a, 1)^{n n+n+p+1}$, only until $p=a-1$. When $p=a$, we can see on other grounds that

and therefore $\quad A_{(n+1)(n+1)}+A_{(n-1)(a+1)}>2 A_{n a+a}$;

but $I$ doubt very much if it is true always that

$$
A_{(n+1)(a+1)+1}+A_{(n-1)(a+1)-1}>2 A_{n a+n} \text {. }
$$

As to $b$ being greater than unity, put $a=3, b=2, n=1, p=3$. 
We can prove at once, by numerical calculation, that the sum of the "advantages" for $\left(\frac{3}{6}+\frac{2}{6}\right)^{2}$ and $\left(\frac{3}{5}+\frac{2}{6}\right)^{8}$, so far from being greater than twice the "advantage" for $\left(\frac{3}{5}+\frac{2}{6}\right)^{6}$, is a negative quantity. Therefore, for this and certain other cases at any rate, $A_{n a+n b+p}+A_{n a+n b-p}$ is not greater than $2 A_{n a+n b}$. I am, in fact, inclined to think that the sum is nearly always negative when $p b$ exceeds by unity a multiple of $a+b$.

32. The above conclusion is very disappointing, as I have becn thereby prevented from pruving our results for evcry case; and, although Art. 4 is demonstrated for all integral values of $b$, it has been necessary to qualify the first paragraph of Art. 1 by the sonicwhat clumsy limitation that $b=1$. There can be little doubt, I think, that the paragraph in question, as well as Art. 30, though necessarily subject to this limitation so far as Art. 28 is concerned, is susceptible of proof by some other method when $b$ is any integer less than $a$. "If an event happen on the average ouce in $m$ times, $m$ being greater than mity, then it is more likely to happen less than once in $m$ times than it is to happen more than once $i .1 m$ times." To my mind, at least, it seems incredible that such a proposition should be true for all integral values of $m$, and not true for all improperfractional values of $m$. Therc the matter must be left at present.

33. It may be interesting to summarize roughly, by means of a figure, the foregoing conchusions. We will confine ourselves to the case when $b=1$. Neglecting higher powers of $\frac{1}{n}$, we may put

$$
\Delta_{n}=h G_{n}-\frac{k}{n} G_{n}
$$

where $h$ is less than $\frac{1}{3}$, and $k$ less than $\frac{1}{15}$. The variations of $G_{n}$, as $n$ changes, are difficult to represent; but we know at least that $G_{n}$ is always less than unity, and continually decreases, $G_{n+1}$ being, roughly speaking, equal to $G_{n}\left(1-\frac{1}{2 n}\right)$; moreover, when $n$ is large, $G_{n}$ varies as $n^{-1}$. Therefore, except when $n$ is very small, we may put

$$
\Delta_{n}=\frac{C}{n^{4}}-\frac{D}{n},
$$

where both $C$ and $D$ are considerably less than unity, and $D$ much smaller than $C$.

Take a horizontal line as axis of $x$, and a vertical line as axis of $y$. When $x$ has any value $r$ : (always integral) let the corresponding value of $y$ (positive or negative) denote the " advantage," at the end of $r$ trials, of a person who gives odds of $a$ to 1 . For values of $a$ 
which are multiples of $a+1$, we thus obtain points on a curve $M M_{1} M_{2} M_{3} \ldots$ above the axis of $x$, always approaching the axis, and convex on the lower side.

When $x$ has any value $n(a+1)+1$, the corresponding value of $y$ will lie between four times and five times its value when

$$
x=n(a+1) \text {; }
$$

ultimately, when $n$ is very large, approaching the former limit. The sum of the ordinates for

$$
x=n(a+1)+1 \text { and } x=n(a+1)-1
$$

will always be exactly double of the ordinate when

$$
x=n(a+1) \text {. }
$$

As $x$ ranges from $n(a+1)+1$ to $n(a+1)+a, y$ will continually diminish; lying, I have reason to think, on a curve of very slight convexity turned downwards, the convexity being, however, so slight that, unless the figure is drawn on a very large scale, it is hardly distinguishable from a straight line. From

$$
x=n(a+1)+a \text { to } x=n(a+1)+a+2 \text {, }
$$

the curve will proceed upwards in an absolutely straight line, and so on, repeating itself in similar fashion for the next period.

For large values of $x$, the highest points of these zigzags will lie very nearly on the curve

$$
y=4 a x^{-1}
$$

and the lowest points very nearly on the curve

$$
y=-2 a x^{-k}
$$

the complete-period curve $M_{1} M I_{2} M I_{3} \ldots$ being

$$
y=a x^{-1}
$$

where $a$ is some positive quantity, a fixed function of $a$.

If it be borne in mind that the vertical proportions are; of necessity, enormously magnified, we may obtain from the subjoined figure a very clear idea of the avernge "advantage" for any given range of trials. If $x$, the number of trials, is known to lie between two values represented on the $x$-line by the points $H$ and $K$, all we have to do is to draw vertical lines $H H^{\prime}$ and $K K^{\prime}$ through $I$ and $K$. The average "advantage " for that range of trials is very nearly obtained by subtracting all the lower shaded areas between $H H^{\prime}$ and $K K^{\prime}$ from all the upper shaded areas between $H H^{\prime}$ and $K K^{\prime},{ }^{*}$ and dividing by the length of $H K$. We perceive at once how, when $H K$ is greater than any complete period-length $P_{1} P_{2}$, the average "advantage" is always

- By an oversight, some of the shading in the figure has been omitted. All the upper triangles ought to be shaded, as well as all the lower ones. 
positive; but that it may be negative when $H K$ is very small and situated close to the left of any of the points $P_{1}, P_{2}, P_{3}$, \&c. We perceive also at once how the average " advantage" diminishes numerically, (i.) if $H K$ is moved bodily to the right, over any distance which is a multiple of $P_{1} P_{2}$; or (ii.) if, $H$ remaining fixed, $K$ is moved to the right over any distance which is a multiple of $P_{1} P_{g}$. These are the main facts which it has been the object of the present paper to prove.

When $x$ is very large, it is interesting to note that any shaded triangle above the axis has clearly four times the area of either adjacent shaded triangle below the axis, which would seem to show, especially when $a$ as well as $x$ is large, that the sum of the positive "advantages" for any complete period, from $x=n(a+1)$ to $n=(n+1)(a+1)$, is four times the sum of the negative "advantages "for the same period.

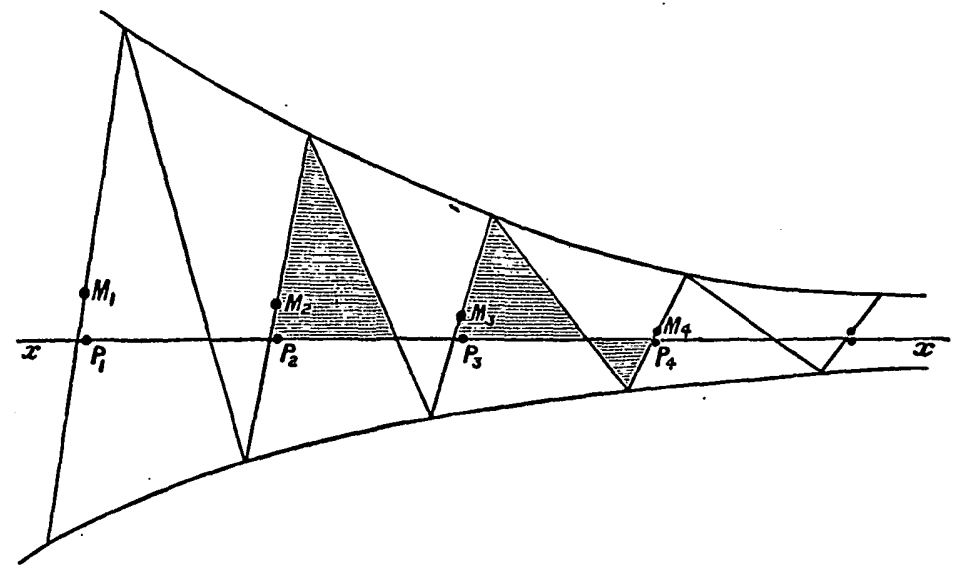

The reader can easily construct for himself the beginning of the "advantage"-curve for any given value of $a$. For instance, when $a=4$, the first thirteen values of $y$, from $x=1$ to $x=13$, are $\cdot 6, \cdot 28$, $\cdot 024,-\cdot 1808, \cdot 06496, \cdot 31072, \cdot 1534, \cdot 00662,-\cdot 12758, \cdot 05361, \cdot 23480$, $\cdot 116690,003294$. Note that here $y$ is positive in every case except when $x=4$ or 9 ; and $I$ have no doubt that in fact $y$ is always positive except when $x$ is of the form $5 m-1$.

Again, when $a=5$ (the case of a die), the first nine values of $y$ are $\cdot 6, \cdot 38, \cdot 148,-0355,-\cdot 1962, \cdot 0717, \cdot 3396, \cdot 2093, \cdot 085$; and I have no doubt that for es:ery complete period there are four positive values of $y$ and two negative ones. The proportions of the curve for these two cases are not quite the same as those in the figure, the latter being illustrative of large values of $x$. 
34. In this and the next three articles, without assuming any of the results of pp. 294-315, we will consider the whole matter from another point of view.

If every term of $(a, b)^{n a+n b}$ be divided by the $(n b+1)^{\text {th }}$ term $G_{n}$, we may, commencing from the middle of the expansion, write, for the short-side and long-side,

$$
\begin{aligned}
& {[S]_{n a+n b}=\frac{n}{n+\frac{1}{a}}+\frac{n\left(n-\frac{1}{b}\right)}{\left(n+\frac{1}{a}\right)\left(n+\frac{2}{a}\right)}+\frac{n\left(n-\frac{1}{b}\right)\left(n-\frac{2}{b}\right)}{\left(n+\frac{1}{a}\right)\left(n+\frac{2}{a}\right)\left(n+\frac{3}{a}\right)}+\ldots} \\
& \quad \text { to } n b \text { terms, } \\
& {[L]_{n a+n b}=\frac{n}{n+\frac{1}{b}}+\frac{n\left(n-\frac{1}{a}\right)}{\left(n+\frac{1}{b}\right)\left(n+\frac{2}{b}\right)}+\ldots \quad \text { to } n a \text { terms. }}
\end{aligned}
$$

If the excess of the former expression over the latter could be expanded in a series of the form $I+C C_{n}^{-1}+D n^{-2}+E n^{-3}+\& c$., all the results of the present paper would be obtained. The fact that

$$
B=\frac{1}{3} \frac{a-b}{a+b} \text {. }
$$

ought, one would think, by putting $n=\infty$, to admit of a fairly simple straightforward proof; I have made repeated attempts, in vain, to obtain one myself.

35. If $s_{r}$ denote the $r^{\text {th }}$ term of the former series, and $l_{r}$ the $r^{\text {th }}$ term of the latter, and if $K_{r}$ denote the ratio of $s_{r}$ to $l_{r}$, we easily obtain

$$
K_{r+1}=\frac{a^{2}}{b^{2}} \cdot \frac{(2 n b+1)^{2}-(2 r+1)^{z}}{(2 n a+1)^{2}-(2 r+1)^{2}} K_{r}
$$

showing that $K_{r}$, which at first is greater than unity, diminishes with increasing rapidity at every subsecpent term, till it becomes zero. As soon, then, as any term $s_{p}$, is less than $l_{p}$, every succeeding shortside term is less than the corresponding long-side term.

Again, if $T_{1}, T_{2}, \ldots T_{n a+n b+1}$ be the successive terms of $(a, b)^{n a+n c}$, and a person bet odds of $a$ shillings to $b$ shillings in a fair wager, after $n a+n b$ trials lis expectation will be

$$
\begin{gathered}
n b(a+b) T_{1}+(n b-1)(a+b) T_{8}+(n b-2)(a+b) T_{8}+\ldots \\
\ldots+(a+b) T_{n b}+0 . T_{n b+1} . \\
-(a+b) T_{n b, 2}-2(a+b) T_{n b+3}-\ldots-n a(a+b) T_{n a+n b+1} .
\end{gathered}
$$


But this expectation is zero. Hence

$$
\begin{aligned}
& T_{n b}+2 T_{n b-1}+\ldots+(n b-1) T_{2}+n b T_{1}=T_{n b+2}+2 T_{n b+3}+\ldots+n a T_{n a+n b+1}, \\
& \text { i.e., } \quad s_{1}+2 s_{9}+3 s_{3}+\ldots+n b . s_{n b}=l_{1}+2 l_{2}+3 l_{3}+\ldots+n a . l_{n a} .
\end{aligned}
$$

36. From the preceding article we can deduce at once that $\Delta_{n}$ is always positive.*

Let $s_{p}$ be the first short-side term which is less than $l_{p}$, the corresponding long-side term. Then, since

and

$$
s_{1}+2 s_{2}+3 s_{y}+\ldots+n b . s_{n b}=l_{1}+2 l_{2}+3 l_{3}+\ldots+n a . l_{n a},
$$

$s_{p}+2 s_{p+1}+3 s_{p+2}+\ldots+(n b-p+1) s_{n b}<l_{p}+2 l_{p+1}+\ldots+(n a-p+1) l_{n a}$,

we have, iy subtraction,

$$
\begin{aligned}
s_{1}+2 s_{3}+\ldots+(p-2) s_{p-2}+ & (p-1)\left(s_{p-1}+\ldots+s_{n b}\right) \\
& >l_{1}+2 l_{2}+\ldots+(p-1)\left(l_{p-1}+\ldots+l_{n a}\right) .
\end{aligned}
$$

But, $s_{1}, s_{2}, \ldots s_{p-1}$ being severally greater than $l_{1}, l_{2}, \ldots l_{p-1}$,

$(p-2) s_{1}+(p-3) s_{2}+\ldots+2 s_{p-3}+s_{p-2}>(p-2) l_{1}+(p-3) l_{s}+\ldots+l_{p-2}$. Therefore, by addition,

$(p-1)\left(s_{1}+s_{q}+\ldots+s_{p-2}+\ldots+s_{n b}\right)>(p-1)\left(l_{1}+l_{g}+\ldots+l_{p-2}+\ldots+l_{n a}\right)$, or

$$
s_{1}+s_{9}+s_{8}+\ldots+s_{n b}>l_{1}+l_{2}+l_{3}+\ldots+l_{n a} \text {. }
$$

37. The desirability of an algebraical proof of the last equation of Art. 35 has been suggested; and I venture to offer the following.

The $(n b)^{\text {th }}$ and $(n b+1)^{\text {th }}$ terns of $(a, b)^{n n+n b-1}$ are both equal to $G_{n}$. Dividing the whole expansion by $G_{n}$, the short-side, in reversed order, may be written

$$
\begin{aligned}
{[S]_{n a+n b-1}=1+\frac{n-\frac{1}{b}}{n+\frac{1}{a}}+} & \frac{\left(n-\frac{1}{b}\right)\left(n-\frac{2}{b}\right)}{\left(n+\frac{1}{a}\right)\left(n+\frac{2}{a}\right)} \\
& +\frac{\left(n-\frac{1}{b}\right)\left(n-\frac{2}{b}\right)\left(n-\frac{3}{b}\right)}{\left(n+\frac{1}{a}\right)\left(n+\frac{2}{a}\right)\left(n+\frac{3}{a}\right)}+\ldots \text { to } n b \text { terms. }
\end{aligned}
$$

- This proof has gradually evolved itself out of some remarks in a letter receired, oubsequently to April 4th, from Dr. Biddle, of Kingston-on-Thames, to whom $\bar{I}$ am in consequence greatly indebted. Dr. Biddle's own proof, which is somewhat different, will appoar in Educational Times Reprint, Vol. LxII., Quest. 12686. 
Subtract from each term the corresponding term in $[S]_{\text {na+n }}$ and wa have

$$
\begin{aligned}
{[S]_{n a+n b-1}-[S]_{n a+n b}=} & \frac{1}{n a} \frac{n}{n+\frac{1}{a}}+\frac{2}{n a} \frac{n\left(n-\frac{1}{b}\right)}{\left(n+\frac{1}{a}\right)\left(n+\frac{2}{a}\right)} \\
& +\frac{3}{n a} \frac{n\left(n-\frac{1}{b}\right)\left(n-\frac{2}{b}\right)}{\left(n+\frac{1}{a}\right)\left(n+\frac{2}{a}\right)\left(n+\frac{3}{a}\right)}+\ldots \\
= & \frac{1}{n a}\left(s_{1}+2 s_{2}+3 s_{3}+\ldots+n b . s_{n b}\right) .
\end{aligned}
$$

But, by Art. 5, $[S]_{n a+n b-1}-[S]_{n a+n b}=\frac{b}{a+b}$;

therefore*

$$
s_{1}+2 s_{2}+3 s_{8}+\ldots+n b \cdot s_{n b}=\frac{n a b}{a+b} .
$$

Similarly,* $\quad l_{1}+2 l_{9}+3 l_{8}+\ldots+n a \cdot l_{n a}=\frac{n a b}{a+b}$;

therefore $s_{1}+2 s_{9}+3 s_{3}+\ldots+n b . s_{n b}=l_{1}+2 l_{2}+3 l_{3}+\ldots+n a . l_{n a}$.

38. The long-side of $(a, b)^{n n+n b-1}$, after division by $G_{n}$, may be writton

$$
[L]_{n a+n b-1}=1+\frac{n-\frac{1}{a}}{n+\frac{1}{b}}+\frac{\left(n-\frac{1}{a}\right)\left(n-\frac{2}{a}\right)}{\left(n+\frac{1}{b}\right)\left(n+\frac{2}{b}\right)}+\ldots \text { to } n a \text { terms. }
$$

Each of the first $n b$ terms of this is greater than the corresponding term in $[S]_{n a+n b-1}$ above. Hence $S_{n a+n b-1}-L_{n a+n b-1}$ is negative, i.e., $A_{n a+n b-1}$ is regative, whatever be the values of $a, b, n$. This proof is of more value than the one given in Art. 25, for it is obtained independently of the $\Delta_{n}$ formula, and leads to the only rigid demonstration I have been able to contrive to show that the function $\psi(a)$ in Art. 9 is absolute zero.

Let us try and put the matter clearly. By Art. $36, A_{n a+n b}$ or $\Delta_{n}$ is always positive; by the present article, $A_{n a+n b-1}$ is always negative; and, by Art. 5 (see also Art. 25), $A_{n a+n b-1}$ always $=A_{n a+n b}-\frac{a-b}{a+b} G_{n}$. Now, if $\psi(a)$ be not absolute zero, it must, for any given value of $a$,

- These two interesting relations have perhaps been proved before; but I am unable to find them anywhere. 
be either positive or negative, and cannot be both. Again, observing the formula of Art. 9, it is evident that the terms following $\psi(a)$, both aloue and when diminished by $\frac{a-b}{a+b} G_{n}$, can be made as small as we please, and therefore smaller than $\psi(a)$, by taking $n$ large enough. Hence, ultimately, $A_{n a+n b}$ and $A_{n a+n b-1}$ are both of the same sign as $\psi(a)$. Therefore, when $n$ is infinite, $\psi(a)$ is both positive and negative; but $\psi(a)$ is independent of $n$, and therefore would be for all values of $n$ both positive and negative, which is inpossible. Therefore $\psi(a)$ must be absolute zero, and $\Delta_{n}$ can contain no term independent of $n$.

39. My task is now completed; but it may be permissible to add three more articles by way of supplement.

The first explorer of a hitherto untrodden region has not always the good fortune to discover the best road at starting; certainly I had not; and an account of the various paths pursued to open out the way in our present subject may be of interest, as saving trouble to future investigators. My only ambition at first was to prove that $\Delta_{n}$ is always positive, the original attempt being by what still seems to me the most natural and straightforward method, i.e., to show that, when $b=1$, the first series in Art. 34 always exceeds the second. Repeated failures in this direction were probably due to lack of skill, for the two series certainly look as if they ought to be manageable, and they may be commended to the reader's attention.

Next, putting $a=2$, the expansion of $(2+1)^{3 n} \times(2+1)^{8}$ was compared with that of $(2+1)^{3 n+3}$, leading to the result

$$
\Delta_{n}-\Delta_{n+1}=\frac{2^{2 n+1}}{3^{3 n+3}} \frac{(3 n) !}{n !(2 n+2) !}(3 n-1) ;
$$

which, finally, after a good deal of trouble, gave $\Delta_{n}=$ the constant term in the expansion of

$$
\frac{y}{y+4}\left\{\frac{4^{n}}{3^{3 n}}(1+y)^{3 n}-1\right\}
$$

which, on making $n$ infinite, is ultimately equal to $\frac{1}{9} \frac{4^{n}}{3^{3 n}}$ times the coefficient of $y^{n}$ in $(1+y)^{3 n}$, or $\frac{1}{6}$ of the greatest term in $\left(\frac{1}{3}+\frac{2}{3}\right)^{3 n}$.

This not only proved $\Delta_{n}$ to be positive when $n$ is infinite, and to increase as $n$ diminishes, which liad been looked for; but it gave the ultimate value of $\Delta_{n}$, which had not been looked for. The same 
method was much more difficult, but still manageable, when applied to $(3+1)^{4 \prime \prime}$; and gave

$$
\Delta_{n}-\Delta_{n+1}=\frac{3^{3 n+1}}{4^{4 n+3}} \frac{(4 n) !}{n !(3 n+3) !}\left(48 n^{2}+14 n-7\right),
$$

positive again; also $\Delta_{n}$ ultimately equal to $\frac{1}{0}$ of the greatest term in $\left(\frac{1}{4}+\frac{3}{4}\right)^{4 n}$. If now $3 n-1$ and $48 n^{2}+14 n-7$ could have becn expressed in the form $\psi(2, n)$, and $\psi(3, n)$, and $\psi$ determined, it would have been allowable to put

$$
\Delta_{n}-\Delta_{n+1}=\frac{4^{4 n+1}}{5^{3 n+4}} \frac{(5 n) !}{n !(4 n+4) !} \psi(4, n)
$$

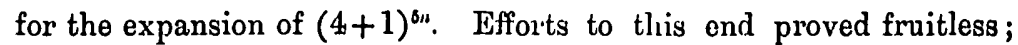
moreover, the direct methol, in its application to $a=4$ and greater values, was utterly unmanageable, and for a long time the work was at a standstill. It seemed clear, however, that, for infinite values of $n$, $\Delta_{n}$ must be always some simple multiple of $G_{n}$.

At length another method was found, which, proving $\Delta_{n}$ ultimately. $=\frac{1}{8} G_{n}$, when $a=4$, and $\mp{ }_{0}^{2} G_{n}$ when $a=5$, suggested the result

$$
\Delta_{n}=\frac{1}{3} \frac{a-1}{a+1} G_{n}
$$

for the general case. The method proved, in fact, applicable to the general case when $n=\infty$, but resolutely refused to prove $\Delta_{n}-\Delta_{n+1}$ always positive when $n$ is finite.

Again work was at a standstill, and recourse was had to a large number of numerical examples, in doubt whether the theorem, after all, was universally true. These examples showing, quite by accident, that $\Delta_{n}$ always lies about half-way between

$$
\frac{1}{3} \frac{a-1}{a+1} G_{n} \text { and } \frac{1}{3} \frac{a-1}{a+1} G_{n+1}
$$

exploration was suggested on a new track. This was to expand $\Delta_{n}$ in a series of ascending powers of $\frac{1}{n}$; which, by a most cumbrous and intricate metlod, gave at length tho second approximation

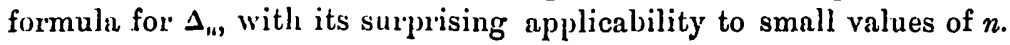
'This, together with the investigation of broken-period sets, formed the basis of the communication on April 4th. The method was still based on the comparison of the two expansions of $(a+1)^{(n+1)(a+1)}$ and $(a+1)^{n(a+1)} \times(a+1)^{a+1}$, and was about tive times the length of the one here given. Moreover, it was utterly inapplicable to the expansion 
of $(a+b)^{n a+n b}$, whoso $\Delta_{n}$ formula was given on that occasion without demonstration.

Not until Mity, i.e., after three months' hard work on the theorem, was it perceived that a fundarnental and yet quite natural mistake had prevailed throughout; which was imagining that tho expansion of $(a+1)^{n(a+1)}$ was moro easily managcable than the expansion of

$$
\left(\frac{a}{a+1}+\frac{1}{a+1}\right)^{n(a+1)}
$$

A new and far shorter method, inapplicable to the former case, was found applicable to the latter, and is the ono here given. By the indulgence of tho Society and the roforees, I have been permitted to entirely re-writo the paper, finally adding Arts. 36 and 38 . It is curious that the apparently simple matter of proving $\psi(a)=0$ should have proved the most baffling task of all ; possibly the reader may be able to devise a simpler and more direct method.

Moreover, we are now able to add the third approximation formula for $\Delta_{n}$, impossible by the former mothod. Arts. 14-19 still seem necessary, even after this addition, so they have been left nearly in their original form.

40. It will be found that the cocflicient of $\frac{1}{n^{3}}$ in the expression for $I^{\prime}$, in Art. 6 is

$$
-\frac{r^{2}(r+1)^{2}}{4.8 a^{3}(a+1)^{8}}\left\{r^{2}+r(8 a+5)+12 a^{2}+16 a+6\right\} .
$$

Summing this from $r=1$ to $r=a$, a very tiresome process, we obtain

$$
\begin{aligned}
S_{(n+1)(a+1)}-S_{n(a+1)} & =\frac{a+2}{6(a+1)}\left[\frac{1}{n}-\frac{1}{n^{2}} \frac{13 a^{2}+21 a+6}{20 a(a+1)}\right. \\
& \left.+\frac{1}{n^{8}} \frac{407 a^{4}+1306 a^{8}+1225 a^{2}+398 a+24}{840 a^{2}(a+1)^{2}}+\ldots\right] G_{n} .
\end{aligned}
$$

Proceeding in Art. 7 to another term, we shall now find that

$$
a_{n+1}=\left\{1-\frac{1}{2 n}+\frac{1}{n^{2}} \frac{11 a^{2}+11 a+2}{24 a(a+1)}-\frac{1}{n^{8}} \frac{7 a^{2}+7 a+2}{16 a(a+1)}+\ldots\right\} G_{n} ;
$$

whence, by an extension of the method of Art. 8, we obtain

$$
\begin{aligned}
S_{n(a+1)}=\frac{1}{2}-\frac{a+2}{3(a+1)}\{ & 1+\frac{2}{45 n} \frac{(a-1)(2 a+1)}{a(a+1)} \\
& \left.-\frac{4}{945 n^{2}} \cdot \frac{(a-1)(2 a+1)\left(a^{2}+a+1\right)}{a^{2}(a+1)^{2}}+\ldots\right\} G_{n} .
\end{aligned}
$$

VOL. XXVI. -No. 520. 
Observing that $945=3^{3} \cdot 5 \cdot 7$, we thus have

$\Delta_{n}=\frac{1}{3} \frac{a-1}{a+1}\left\{1-\frac{1}{n} \frac{2^{2}(a+2)(2 a+1)}{3^{2} \cdot 5 a(a+1)}\right.$

$$
\left.+\frac{1}{n^{2}} \frac{2^{3}(a+2)(2 a+1)\left(a^{9}+a+1\right)}{3^{3} \cdot 5 \cdot 7 a^{2}(a+1)^{2}}+\ldots\right\} G_{n} \text {. }
$$

This may be compared with the two expressions for $\Delta_{n}-\Delta_{n+1}$ given in Art. 39. The reader, by substituting numerically, will moreover find the "proportion of error" now, generally speaking, about $\frac{1}{4}$ of what it was in the second approximation, and again less for large than for small values of $a$. It is disappointing to find, however, that this third approximation is, in all the cases I have tried, less than the actual value, suggesting the presumption that the coefficient of $\frac{1}{n^{8}}$ will be again positive, and destroying, in one detail, the symmetry of the series.

I have since proved, by an independent method, that, when $a=2$, the next term inside the bracket is $+\frac{56}{3^{7} \cdot n^{3}}$, and, when $a=3$, it is $+\frac{169}{4.3^{7} \cdot n^{8}}$. Both of these results conform with the formula

$$
+\frac{1}{n^{3}} \frac{2^{4}(a+2)(2 a+1)\left(a^{3}+a+1\right)^{9}}{3^{4} \cdot 5 \cdot 7 \cdot a^{8}(a+1)^{8}}
$$

suggesting the possibility of this:being, for all values of $a$, the next term inside the bracket. On testing numerically, I find this fourth approximation, both for $a=2$ and $a=3$, greater than the actual value of $\Delta_{m}$, implying apparently that the coefficient of $\frac{1}{n^{4}}$ will be for those cases, and therefore also for the general case, negative.

It is interesting to note that the quantities $1,-\frac{2^{3}}{3^{3} .5}, \frac{2^{4}}{3^{3} .5 .7}$, $\frac{2^{5}}{3^{4} .5 .7}$ form a converging series; so that, if the above formula gives the actual coefficient of $\frac{1}{n^{3}}$, a still stronger presumption is afforded of the correctness of the hypothesis of Art. 19.

The regularity of form of the successive coefficients further suggests the possibility of expanding either of the series of Art. 34, and their difference, in such manner as to obtain an expression for the general term. Whether, in fact, our theorem and formula admit of a simple, straightforward proof, or remain for generations 
apparently incapable of it, like Bernoulli's Theorem, will be left for the future to decide.

41. In conclusion, it is hardly necessary to assure the reader that my object has been in no sense to prove that the gambler who gives odds is in a more advantageous position than the one who takes odds. In fact, I have proved afresh, in Art. 37, that in a fair wager the gambler's "expectation" is always zero, whether after 10 trials or after 10,000,000. The vocabulary of the English language, and probably of all languages, is strikingly deficient in words exactly suitable for the scientific treatment of probability; and the word "advantage," to denote excess of probability of net gain over probability of net loss, is the best I could devise. But the fact that it has always been carefully guarded within inverted commas may suffice to show that it is throughout employed in a purely technical sense, and not in the ordinary one.

Thursday, May 9th, 1895.

Major P. A. MACMAHON, P.A., F.R.S., President, in the Chair.

Mr. William Henry Metzler, A.B. (Toronto), Associate-Professor, Syracuse University, Syracuse, New York, and Mr. Frederick William Russell, B.A., formerly Scholar of Trinity College, Cambridge, assistant-nuaster in University College School, London, were elected members. Mr. P. H. Cowell was admitted into the Society.

The following communications were made :-

On the most General Solution of Given Degree of Laplace's Equation: Dr. Hobson.

A Property of a Skew-Determinant, and on the Geometrical Meaning of a Form of the Orthogonal Substitution: Prof. M. J. M. Hill.

The Spherical Catenary: Prof. Greenhill and Mr. T. I. Dewar (a model was exhibited of this catenary, formed by a chain wrapped on a terrestrial globe). 
Mr. Jeppel exhilited a set of Napier's Bones, of date 1746, and explaincd how they were used in calculation.

The following papers were takon as read:

On those Orthogonal Sulstitutions that can be generated by the Repetition of an Infinitesimal Orthogonal Substitution : Dr. H. T'aber.

Notes on the Theory of Groups of Finite Order (continuation): Prof. W. Burnside.

Applications of Trigrapliy: Mr. J. W. Russell.

The Reciprocators of 'Two Conics: Messrs. J. W. Russell and A. Ti. Jolliffe.

The following presents were received:-

Queen's College, Galway, "Calendar fưr 1894-95," 8ro ; Dublin, 1895.

"Boibliitter zu den Annalon dor Physik und Chemie," Bd. xrx., St. 4 ; Leipzig, 1895.

"Cumbridgro Philosophicnl Society, Procecdings," Vol. vrrr., Pt. 1 ; October, - 1894.

"Vicrtoljahrsschrift dor Naturforschenden Gesellschaft in Zürich," Jahrgang 40, II oft $1 ; 1895$.

Kluyver, J. C.-“Invarianten-Theorie," pamph., 8vo (offprint).

"Borichto über die Verhandlungen der Königlich Sächsischen Gesollschaft dor Wisscukchafton zu Luipzig," IIr., 1894.

"Archives Néerlandaires," Tome xxux., Liv. I ; Harlem, 1895.

"Procecelings of the Royal Socicty," Vol. J.vir., Nos. 343-344.

"Proccedinge of the Physical Socioty of London," Nos. 65, 56; April-May, 1895.

" Bulletin of tho Americnn Mathematicnl Society," 2nd Scries, Vol. r., Nos. 6-7. New York.

"Société des Antiqunires do l'Ouest-Bullotin," 'Trim. Iv. ; 1894.

"Bulletin des Sciences Mathématiques," Séric 2, Tomo xux., Avril, 1895 ; Paris.

"Rendiconto dell' Accademia della Scienzo Fisicho e Matematiche," Scrie 3", Vol. I., Fine. 3 ; Nipoli, 1895.

"Mémoiros de la Sucićté. des Sciences do Bordeaux," Tome III., Cahicr 2; Tome 17., Cahier 1, 2 ; Pris, 1893.

Porot, $\Lambda$.- "Sur l'Existenco et la Propagation des Oscillations Electro-mugnétiques dens l' $\Lambda$ ir," pamph.

Ruyct, G.-Observations Pluviométriques et Thermomótriques, 8vo; Bordcaux, 1893.

Thonue, J.-“Untersuchungen ïber zweizweidentige Verwandschnften und einige Frzeugnisac derwellen," roy. 8ro ; Loipzig, 1895.

"Educntional Times," Mruy, 1895.

" Atti della Realo Accademia doi Lincoi-Rendiconti," 1 Sem., Vol. xv., Fase. 6-7; Rown, 1895.

"Annales do In Facultó des Sciences do Mrarseille," Tome rv., Fasc. 1, 2, 3.

"Journal de l'Ecole Polytechnique," Calicr 64; Paris, 1894. 
"Indian Engineering," Vol. xvrr., Nos. 11-15.

“Acta Mathematica," xrx., No. 2.

"The Mathematical Magazine," edited by Dr. A. Martin, Washington, D.C., 1895 ; Vol. Ir., No. 9, Janunry, 1895.

"List of Merabers of the London Mathematical Socioty," from January 15th, 1866 , to November 9th, 1893 ; 30 sessions (14th November, 1867, called 4th session, 2 previous lista).

Notes on the Theory of Groups of Finite Order (continued). By W. Burnside. Received May 7th, 1895. Read May 9th, 1895.

The first of the two notes in the present communication deals with certain properties of groups whose order is even. It is shown that if $2^{m}$ is the highest power of 2 contriued in the order of a group, and if the sub-groups of order $2^{m}$ are cyclical, the group cannot be simple; so that, in particular, no group whose order is divisible by 2 , but not by 4, can be simple. When the highest power of 2 which divides the order of a group is either $2^{2}$ or $2^{3}$ it is shown that, unless the group contrins a smaller number of distinct conjugate sets of operations of orlers 2 or 4 than the sub-groups of orders $2^{3}$ and $2^{3}$ respectively contain, the group cannot be simple. In the first case, this condition cannot be satisfied unless 3 is a factor of the order; nor can it be satisfied in the second case unless either 3 or 7 is a factor of the order, and, therefore, no group of eren order can be simple unless its orcler is divisible by 12,16 , or 56 . It secms extrenely probable that this property may be extended to the more general form that, if the order of a group be

$$
N=2^{m} n
$$

where $n$ is odd, and if $N$ is relatively prime to $2^{m}-1,2^{n-1}-1, \ldots 2^{2}-1$, the group cannot be simple; but I have not hitherto succeeded in proving this more general result.

In the second note, Dr. Cole's and Herr Hölder's determination of all simple groups whose orders do not exceed 660 is carried on from 660 to 1092 , the order of the next known simple group, with the result of showing that no simple groups exist in the interval. 